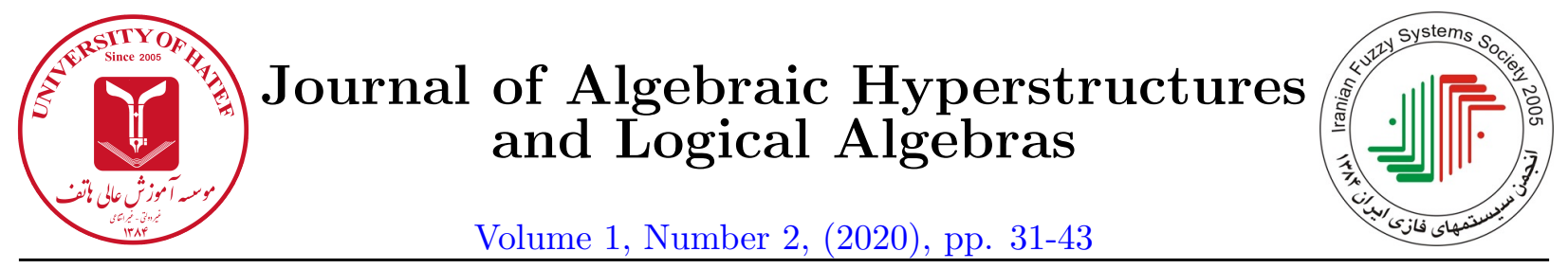

\title{
Fuzzy congruence relations on pseudo BE-algebras
}

\author{
A. Rezaei ${ }^{1}$, A. Borumand Saeid ${ }^{2}$ and Q. Zhan ${ }^{3}$ \\ ${ }^{1}$ Department of Mathematics, Payame Noor University, P.o.box. 19395-3697, Tehran, Iran \\ ${ }^{2}$ Department of Pure Mathematics, Faculty of Mathematics and Computer, Shahid Bahonar University of \\ Kerman, Kerman, Iran \\ ${ }^{3}$ Department of Mathematics, Beihang University (BUAA), BUA-Haidian School of Applied Technology, \\ china
}

rezaei@pnu.ac.ir, arsham@uk.ac.ir, by1709120@buaa.edu.cn

\begin{abstract}
In this paper, we introduce the concept of fuzzy congruence relations on a pseudo BE-algebra and some of properties are investigated. We show that the set of all fuzzy congruence relations is a modular lattice and the quotient structure induced by fuzzy congruence relations is studied.
\end{abstract}

\section{Article Information}

Corresponding Author:

A. Borumand Saeid;

Received: April 2020;

Accepted: Invited paper.

Paper type: Original.

Keywords:

(pseudo) BE-algebra, (fuzzy) congruence relation, (fuzzy) medial filter.

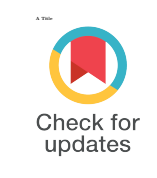

\section{Introduction}

The notion of a BE-algebra was introduced by H.S. Kim et al. 77. A. Borumand Saeid et al. introduced some types of filters in BE-algebras [1. Since developing algebraic models for noncommutative multiple-valued logics is a central topic in the study of fuzzy systems. R.A. Borzooei et al. generalized the notion of BE-algebras and introduced the notion of pseudo BE-algebras, pseudo subalgebras, pseudo filters and investigated some related properties [3]. A. Rezaei et al. introduced the notion of distributive pseudo BE-algebras and normal pseudo filters and proved some basic properties. They showed that in distributive pseudo BE-algebras normal pseudo filters and pseudo filters coincide [4]. L.C. Ciungu introduced the notion of commutative pseudo BEalgebras and proved that the class of commutative pseudo BE-algebras is term equivalent to the class of commutative pseudo BCK-algebras [5].

https://doi.org/10.29252/HATEF.JAHLA.1.2.4 
L.A. Zadeh introduced the notion of fuzzy sets and fuzzy relations [18. Then many authors have studied about it. K.J. Lee defined the notion of ideals in pseudo BCI-algebras [9]. Fuzzy ideals of pseudo BCK-algebras were investigated in [6. Also, A. Walendziak et al. consider fuzzy ideals theory in pseudo BCH-algebras and provided conditions for a fuzzy set to be a fuzzy ideal [17]. Since then V. Murali have studied fuzzy congruence relations on algebras [10, 11]. Further, M. Kondo has defined a fuzzy congruence relation on a group and showed that there is a lattice isomorphism between the set of fuzzy normal subgroups of a group and the set of fuzzy congruences on this group [8]. R.A. Borzooei et al. introduced the concept of a fuzzy filter of a BL-algebra, with respect to a t-norm and proved that there is a correspondence bijection between the set of all $\mathrm{T}$ fuzzy filters of a BL-algebra and the set of all T-fuzzy congruences relations in that BL-algebra [2]. Recently, A. Rezaei et al. discussed on (fuzzy) congruence relations in (pseudo) CI/BE-algebras and studied some of their properties [13, 14, 15].

In this paper, since congruence relations are one of the important concept in algebraic structure, motivated by it, we apply the notion of fuzzy congruence relations on pseudo BE-algebras and discuss on the quotient algebras via this congruence relations. We show that quotient of a pseudo BE-algebra via a fuzzy congruence relation is a pseudo BE-algebra. Moreover, we show that in a distributive pseudo BE-algebra $X$ for every fuzzy medial filter $\bar{\mu}$ there is a fuzzy congruence relation $\bar{\theta}$ such that $\bar{\theta}_{1}=\bar{\mu}$.

\section{Preliminaries}

In this section, we review the basic definitions and some elementary aspects that are necessary for this paper.

Definition 2.1. 3] An algebra $(X ; \rightarrow, \rightsquigarrow, 1)$ of type $(2,2,0)$ is called a pseudo BE-algebra if for all $x, y, z \in X$, it satisfies the following axioms:

$\left(\mathrm{psBE}_{1}\right) x \rightarrow x=x \rightsquigarrow x=1$,

$\left(\mathrm{psBE}_{2}\right) x \rightarrow 1=x \rightsquigarrow 1=1$,

$\left(\mathrm{psBE}_{3}\right) 1 \rightarrow x=1 \rightsquigarrow x=x$,

$\left(\mathrm{psBE}_{4}\right) x \rightarrow(y \rightsquigarrow z)=y \rightsquigarrow(x \rightarrow z)$,

$\left(\mathrm{psBE}_{5}\right) x \rightarrow y=1$ if and only if $x \rightsquigarrow y=1$.

In a pseudo BE-algebra $(X ; \rightarrow, \rightsquigarrow, 1)$, for all $x, y \in X$, one can introduce a binary relation $\leq$ on $X$ by

$$
x \leq y \Longleftrightarrow x \rightarrow y=1 \Longleftrightarrow x \rightsquigarrow y=1 .
$$

Remark 2.2. If a pseudo BE-algebra $X$ satisfies $x \rightarrow y=x \rightsquigarrow y$, for all $x, y \in X$, then $X$ is called a BE-algebra.

Proposition 2.3. [3] In a pseudo BE-algebra $X$, the following statements hold:

(i) $x \rightarrow(y \rightsquigarrow x)=1, x \rightsquigarrow(y \rightarrow x)=1$,

(ii) $x \rightsquigarrow(y \rightsquigarrow x)=1, x \rightarrow(y \rightarrow x)=1$,

(iii) $x \rightsquigarrow[(x \rightsquigarrow y) \rightarrow y]=1, x \rightarrow[(x \rightarrow y) \rightsquigarrow y]=1$,

(iv) $x \rightarrow[(x \rightsquigarrow y) \rightarrow y]=1, x \rightsquigarrow[(x \rightarrow y) \rightsquigarrow y]=1$,

(v) if $x \leq y \rightarrow z$, then $y \leq x \rightsquigarrow z$, 
(vi) if $x \leq y \rightsquigarrow z$, then $y \leq x \rightarrow z$,

(vii) $1 \leq x$ implies $x=1$,

(viii) if $x \leq y$, then $x \leq z \rightarrow y$ and $x \leq z \rightsquigarrow y$, for all $x, y, z \in X$.

Definition 2.4. [4] A pseudo BE-algebra $X$ is said to be distributive, if it satisfies only one of the following conditions:

$\left(\mathrm{D}_{1}\right) x \rightarrow(y \rightsquigarrow z)=(x \rightarrow y) \rightsquigarrow(x \rightarrow z)$,

$\left(\mathrm{D}_{2}\right) x \rightsquigarrow(y \rightarrow z)=(x \rightsquigarrow y) \rightarrow(x \rightsquigarrow z)$, for all $x, y, z \in X$.

Note that if $(X ; \rightarrow, \rightsquigarrow, 1)$ is a pseudo BE-algebra, then $(X ; \rightsquigarrow, \rightarrow, 1)$ is a pseudo BE-algebra, too. By [4, Theorem 2], if $X$ satisfies $\left(D_{1}\right)$ and $\left(D_{2}\right)$, then $\rightarrow=\rightsquigarrow$.So, in this paper, every distributive pseudo BE-algebra satisfies $\left(\mathrm{D}_{1}\right)$.

Also, note that if $x \rightarrow(z \rightsquigarrow y)=x \rightsquigarrow(z \rightarrow y)$, for all $x, y, z \in X$, then $\rightarrow=\rightsquigarrow$, since if $z:=1$ and using $\left(\mathrm{psBE}_{3}\right)$, we get

$$
x \rightarrow y=x \rightarrow(1 \rightsquigarrow y)=x \rightsquigarrow(1 \rightarrow y)=x \rightsquigarrow y .
$$

Definition 2.5. [16] A fuzzy set $\bar{\mu}$ of $X$ is called a fuzzy filter, if for all $x, y \in X$, it satisfies the following conditions:

$$
\begin{aligned}
& \left(\mathrm{FF}_{1}\right) \bar{\mu}(1) \geq \bar{\mu}(x), \\
& \left(\mathrm{FF}_{2}\right) \bar{\mu}(y) \geq \min [\bar{\mu}(x), \bar{\mu}(x \rightarrow y)] .
\end{aligned}
$$

Definition 2.6. [12] A fuzzy set $\bar{\mu}$ of $X$ is called a fuzzy medial filter if for all $x, y, z \in X$, it satisfies $\left(\mathrm{FF}_{1}\right)$ together with the following conditions:

$\left(\mathrm{FMF}_{1}\right) \bar{\mu}(x \rightarrow y) \geq \min [\bar{\mu}(x \rightarrow z), \bar{\mu}(z \rightarrow y)]$

$\left(\mathrm{FMF}_{2}\right) \bar{\mu}(x \rightsquigarrow y) \geq \min [\bar{\mu}(x \rightsquigarrow z), \bar{\mu}(z \rightsquigarrow y)]$.

Note. From now on, $X$ denote a pseudo BE-algebra, unless otherwise is stated.

\section{Fuzzy congruence relations in pseudo BE-algebras}

In this section, we discussed the basic properties of fuzzy congruence relations on pseudo BEalgebras. Let $X$ be a pseudo BE-algebra. A fuzzy relation $\bar{\theta}$ on $X$ is a map $\bar{\theta}: X \times X \rightarrow[0,1]$.

Definition 3.1. A fuzzy relation $\bar{\theta}$ is called a fuzzy congruence relation on $X$ if it satisfies the following conditions: for all $x, y, z, u \in X$,

$\left(\mathrm{FC}_{1}\right) \bar{\theta}(x, x)=\bar{\theta}(1,1)$,

$\left(\mathrm{FC}_{2}\right) \bar{\theta}(x, y)=\bar{\theta}(y, x)$,

$\left(\mathrm{FC}_{3}\right) \bar{\theta}(x, z) \geq \sup _{y \in X} \min [\bar{\theta}(x, y), \bar{\theta}(y, z)]$

$\left(\mathrm{FC}_{4}\right) \bar{\theta}(x \rightarrow u, y \rightarrow u) \geq \bar{\theta}(x, y)$ and $\bar{\theta}(x \rightsquigarrow u, y \rightsquigarrow u) \geq \bar{\theta}(x, y)$ (right compatible), 
$\left(\mathrm{FC}_{5}\right) \bar{\theta}(u \rightarrow x, u \rightarrow y) \geq \bar{\theta}(x, y)$ and $\bar{\theta}(u \rightsquigarrow x, u \rightsquigarrow y) \geq \bar{\theta}(x, y)$ (left compatible).

Let $\mathrm{FCon}(\mathrm{X})$ denote the set of all fuzzy congruence relations on $X$.

Example 3.2. Let $X=\{1, a, b, c\}$ and the binary operations $\rightarrow$ and $\rightsquigarrow$ defined as follows:

\begin{tabular}{c|cccc}
$\rightarrow$ & 1 & $a$ & $b$ & $c$ \\
\hline 1 & 1 & $a$ & $b$ & $c$ \\
$a$ & 1 & 1 & $a$ & 1 \\
$b$ & 1 & 1 & 1 & 1 \\
$c$ & 1 & $a$ & $a$ & 1
\end{tabular}

\begin{tabular}{l|llll}
$\rightsquigarrow$ & 1 & $a$ & $b$ & $c$ \\
\hline 1 & 1 & $a$ & $b$ & $c$ \\
$a$ & 1 & 1 & $c$ & 1 \\
$b$ & 1 & 1 & 1 & 1 \\
$c$ & 1 & $a$ & $b$ & 1
\end{tabular}

Then $(X ; \rightarrow, \rightsquigarrow, 1)$ is a pseudo BE-algebra. Define $\bar{\theta}: X \times X \rightarrow[0,1]$ as follows:

\begin{tabular}{c|cccc}
$\bar{\theta}$ & 1 & $a$ & $b$ & $c$ \\
\hline 1 & 0.87 & 0.32 & 0.32 & 0.32 \\
$a$ & 0.32 & 0.87 & 0.32 & 0.32 \\
$b$ & 0.32 & 0.32 & 0.87 & 0.32 \\
$c$ & 0.32 & 0.32 & 0.32 & 0.87
\end{tabular}

Then $\bar{\theta}$ is a fuzzy congruence relation on $X$.

Lemma 3.3. If $\bar{\theta}$ satisfies $\left(\mathrm{FC}_{2}\right),\left(\mathrm{FC}_{3}\right)$ and $\left(\mathrm{FC}_{4}\right)$, then $\left(\mathrm{FC}_{1}\right)$ is equivalent to

$$
\bar{\theta}(1,1) \geq \bar{\theta}(x, y) \text {, for all } x, y \in X .
$$

Proof. Assume that $\bar{\theta}(1,1)=\bar{\theta}(x, x)$. Since $\bar{\theta}$ satisfies $\left(\mathrm{FC}_{2}\right)$ and $\left(\mathrm{FC}_{3}\right)$, we have

$$
\bar{\theta}(1,1)=\bar{\theta}(x, x) \geq \sup _{y \in X} \min [\bar{\theta}(x, y), \bar{\theta}(y, x)]=\sup _{y \in X} \bar{\theta}(x, y) \geq \bar{\theta}(x, y) .
$$

Conversely, using $\left(\mathrm{FC}_{4}\right)$ we get $\bar{\theta}(x, x)=\bar{\theta}(1 \rightarrow x, 1 \rightarrow x) \geq \bar{\theta}(1,1)$. On the other hand, since $\bar{\theta}(x, x) \leq \bar{\theta}(1,1)$, we get $\bar{\theta}(x, x)=\bar{\theta}(1,1)$.

Proposition 3.4. If $\bar{\theta} \in \mathrm{FCon}(X)$, then for all $x, y \in X$

$$
\bar{\theta}(x, y) \leq \bar{\theta}(x \rightarrow y, 1)=\bar{\theta}(x \rightsquigarrow y, 1) .
$$

Proof. Let $x, y \in X$. Using $\left(\mathrm{FC}_{4}\right)$ and $\left(\mathrm{psBE}_{1}\right)$, we have

$$
\bar{\theta}(x, y) \leq \bar{\theta}(x \rightarrow y, y \rightarrow y)=\bar{\theta}(x \rightarrow y, 1) .
$$

Applying $\left(\mathrm{FC}_{4}\right),\left(\mathrm{FC}_{5}\right),\left(\mathrm{psBE}_{4}\right),\left(\mathrm{psBE}_{1}\right)$ and $\left(\mathrm{psBE}_{2}\right)$ we get

$$
\begin{aligned}
\bar{\theta}(x \rightarrow y, 1) & \leq \bar{\theta}((x \rightarrow y) \rightarrow y, 1 \rightarrow y) \leq \bar{\theta}(x \rightsquigarrow((x \rightarrow y) \rightarrow y), x \rightsquigarrow(1 \rightarrow y)) \\
& =\bar{\theta}((x \rightarrow y) \rightsquigarrow(x \rightarrow y), 1 \rightarrow(x \rightsquigarrow y))=\bar{\theta}(1, x \rightsquigarrow y)
\end{aligned}
$$

Thus, $\bar{\theta}(x \rightarrow y, 1) \leq \bar{\theta}(1, x \rightsquigarrow y)=\bar{\theta}(x \rightsquigarrow y, 1)$. By a similar argument $\bar{\theta}(x \rightsquigarrow y, 1) \leq \bar{\theta}(1, x \rightarrow$ $y)=\bar{\theta}(x \rightarrow y, 1)$. Therefore, $\bar{\theta}(x \rightarrow y, 1)=\bar{\theta}(x \rightsquigarrow y, 1)$.

Note that, in the Proposition 3.4 if $\bar{\theta}(x, y)=\bar{\theta}(x \rightarrow y, 1)$, for all $x, y \in X$, then $\bar{\theta}(x, y)=$ $\bar{\theta}(1,1)$. Using $\left(\operatorname{psBE}_{3}\right)$, we have $\bar{\theta}(x, 1)=\bar{\theta}(x \rightarrow 1,1)=\bar{\theta}(1,1)$, and so, for all $x \in X$, we have $\bar{\theta}(x, 1)=\bar{\theta}(1,1)$. Since $x \rightarrow y \in X$, we obtain $\bar{\theta}(x \rightarrow y, 1)=\bar{\theta}(1,1)$. Thus, $\bar{\theta}(x, y)=\bar{\theta}(1,1)$. 
Proposition 3.5. Let $\bar{\theta}, \bar{\eta} \in \mathrm{FCon}(X)$. Then $\bar{\theta} \cap \bar{\eta} \in \mathrm{FCon}(X)$, where

$$
(\bar{\theta} \cap \bar{\eta})(x, y)=\min [\bar{\theta}(x, y), \bar{\eta}(x, y)] .
$$

Remark 3.6. However, the following example shows that union of two fuzzy congruence relation $\bar{\theta}$ and $\bar{\eta}$ is not necessarily a fuzzy congruence relation.

Example 3.7. Let $X=\{1, a, b\}$. Define the binary operation $\rightarrow$ on $\mathrm{X}$ as follows:

\begin{tabular}{l|lll}
$\rightarrow$ & 1 & $a$ & $b$ \\
\hline 1 & 1 & $a$ & $b$ \\
$a$ & 1 & 1 & $b$ \\
$b$ & 1 & 1 & 1
\end{tabular}

Then $(X ; \rightarrow, 0)$ is a BE-algebra. If put $\rightsquigarrow:=\rightarrow$, then $(X ; \rightarrow, \rightsquigarrow, 0)$ is a pseudo BE-algebra. Define the fuzzy relations $\bar{\theta}$ and $\bar{\eta}$ as follows:

\begin{tabular}{|c|c|c|c|c|c|c|c|c|}
\hline $\bar{\theta}$ & 1 & $a$ & $b$ & \multirow{4}{*}{ and } & $\bar{\eta}$ & 1 & $a$ & $b$ \\
\hline 1 & 0.52 & 0.42 & 0.42 & & 1 & 0.52 & 0.3 & 0.3 \\
\hline$a$ & 0.42 & 0.52 & 0.42 & & $a$ & 0.3 & 0.52 & 0.3 \\
\hline$b$ & 0.42 & 0.42 & 0.52 & & $b$ & 0.3 & 0.3 & 0.52 \\
\hline
\end{tabular}

Then $(\bar{\theta} \cup \bar{\eta})(x, y)=\max [\bar{\theta}(x, y), \bar{\eta}(x, y)]$ is not a fuzzy congruence relation on $X$.

Definition 3.8. Let $\bar{\theta}, \bar{\eta} \in \mathrm{FCon}(X)$. Define the composition $\bar{\theta} \circ \bar{\eta}$ by:

$$
(\bar{\theta} \circ \bar{\eta})(x, y)=\sup _{z \in X} \min [\bar{\theta}(x, z), \bar{\eta}(z, y)] .
$$

Example 3.9. Consider the pseudo BE-algebra given in Example 3.2. Define fuzzy congruence relations $\bar{\theta}$ and $\bar{\eta}$ as follows:

\begin{tabular}{l|lllll|lllll}
$\bar{\theta}$ & 1 & $a$ & $b$ & $c$ & & $\bar{\eta}$ & 1 & $a$ & $b$ & $c$ \\
\hline 1 & 0.75 & 0.44 & 0.44 & 0.44 \\
$a$ & 0.44 & 0.75 & 0.44 & 0.44 \\
$b$ & 0.44 & 0.44 & 0.75 & 0.44 & & & & & & \\
& & & 0.65 & 0.24 & 0.24 & 0.24 \\
$c$ & 0.44 & 0.44 & 0.44 & 0.75 & & $a$ & 0.24 & 0.65 & 0.24 & 0.24 \\
& & $b$ & 0.24 & 0.24 & 0.65 & 0.24 \\
& & & 0.24 & 0.24 & 0.24 & 0.65
\end{tabular}

Then $\bar{\theta} \circ \bar{\eta}$ is a fuzzy congruence relation on $X$ by the following table.

\begin{tabular}{l|llll}
$\bar{\theta} \circ \bar{\eta}$ & 1 & $a$ & $b$ & $c$ \\
\hline 1 & 0.65 & 0.44 & 0.44 & 0.44 \\
$a$ & 0.44 & 0.65 & 0.44 & 0.44 \\
$b$ & 0.44 & 0.44 & 0.65 & 0.44 \\
$c$ & 0.44 & 0.44 & 0.44 & 0.65
\end{tabular}

By induction, we have:

Theorem 3.10. Let $\bar{\theta} \in \mathrm{FCon}(X)$. Then $\bigcup_{n=1}^{\infty} \bar{\theta}^{n}$ is so, where, $\bar{\theta}^{n}=\bar{\theta} \circ \bar{\theta} \circ \ldots \circ \bar{\theta}$. 
Theorem 3.11. $(\mathrm{FCon}(X), \subseteq)$ is a complete lattice, where $\subseteq$ is defined by:

$$
\bar{\theta} \subseteq \bar{\eta} \text { if and only if } \bar{\theta}(x, y) \leq \bar{\eta}(x, y) \text {, for all } x, y \in X .
$$

Proof. Clearly $\subseteq$ is a partial order relation. It is easy to check that the relation $\bar{\sigma}$ defined by $\bar{\sigma}(x, y)=1$, for all $x, y \in X$ is in $\mathrm{FCon}(\mathrm{X})$ and the relation $\bar{\lambda}$ defined by $\bar{\lambda}(x, x)=\bar{\lambda}(1,1)$, for all $x \in X$ and $\bar{\lambda}(x, y)=0$ for $x \neq y$ is in $\mathrm{FCon}(\mathrm{X})$. Also, $\bar{\sigma}$ is the greatest element and $\bar{\lambda}$ is the least element of $\mathrm{FCon}(\mathrm{X})$ w.r.t. $\subseteq$. Let $\left\{\bar{\theta}_{i}\right\}_{i \in I}$ be a non-empty collection of fuzzy congruence relations in $\mathrm{FCon}(\mathrm{X})$. Let $\bar{\theta}(x, y)=\inf _{i \in X} \bar{\theta}_{i}(x, y)$, for all $x, y \in X$. It is easy to see that $\left(\mathrm{FC}_{1}\right),\left(\mathrm{FC}_{2}\right)$, $\left(\mathrm{FC}_{3}\right),\left(\mathrm{FC}_{4}\right)$ and $\left(\mathrm{FC}_{5}\right)$. Also, we have

$$
\begin{aligned}
& \bar{\theta} \circ \bar{\theta}(x, y)=\sup _{z \in X} \min \{\bar{\theta}(x, z), \bar{\theta}(z, y)\} \\
& =\sup _{z \in X} \min \left\{\inf _{i \in I} \bar{\theta}_{i}(x, z), \inf _{i \in I} \bar{\theta}_{i}(z, y)\right\} \\
& =\sup _{z \in X} \inf _{i \in I}\left\{\min \left[\bar{\theta}_{i}(x, z), \bar{\theta}_{i}(z, y)\right]\right\} \\
& \leq \inf _{i \in I} \sup _{z \in X}\left\{\min \left[\bar{\theta}_{i}(x, z), \bar{\theta}_{i}(z, y)\right]\right\} \\
& =\inf \left(\bar{\theta}_{i} \circ \bar{\theta}_{i}\right)(x, y) \\
& \leq \inf _{i \in I} \bar{\theta}_{i}(x, y) \\
& =\bar{\theta}(x, y) \text {. }
\end{aligned}
$$

That is, $\bar{\theta} \in \mathrm{FCon}(X)$. Since $\bar{\theta}$ is the greatest lower bound of $\left\{\bar{\theta}_{i}\right\}_{i \in I}$, hence $(\mathrm{FCon}(X), \subseteq)$ is a complete lattice.

Theorem 3.12. $(\mathrm{FCon}(X), \subseteq)$ is a modular lattice.

Proof. Assume that $\bar{\theta}, \bar{\eta}, \bar{\zeta} \in \mathrm{FCon}(X)$ and $\bar{\theta} \subseteq \bar{\zeta}$. It is sufficient to prove that $(\bar{\theta} \circ \bar{\eta}) \cap \bar{\zeta} \subseteq \bar{\theta} \circ(\bar{\eta} \cap \bar{\zeta})$. For every $(x, y) \in X \times X$ and $z \in X$, since $\min \{\bar{\theta}(x, z), \bar{\zeta}(x, z)\}=\bar{\theta}(x, z)$, applying $\left(\mathrm{FC}_{3}\right)$ for $\bar{\zeta}$, we get

$$
\begin{aligned}
{[(\bar{\theta} \circ \bar{\eta}) \cap \bar{\zeta}](x, y) } & =\min _{[}[(\bar{\theta} \circ \bar{\eta})(x, y), \bar{\zeta}(x, y)] \\
& =\min [\sup \min \{\bar{\theta}(x, z), \bar{\theta}(z, y)\}, \bar{\zeta}(x, y)] \\
& =\sup _{z \in X}\{\min [\bar{\theta}(x, z), \bar{\theta}(z, y)], \bar{\zeta}(x, y)\} \\
& =\sup _{z \in X}\{\min [\bar{\theta}(x, z), \bar{\theta}(z, y)], \bar{\zeta}(x, z), \bar{\zeta}(x, y)\} \\
& \leq \sup _{z \in X}\{\min [\bar{\theta}(x, z), \bar{\theta}(z, y)], \bar{\zeta}(z, y)\} \\
& =\sup _{z \in X}\{\bar{\theta}(x, z), \min [\bar{\theta}(z, y), \bar{\zeta}(z, y)]\} \\
& =\sup _{z \in X}\{\bar{\theta}(x, z),[\bar{\theta} \cap \bar{\zeta}](z, y)\} \\
& =[\bar{\theta} \circ(\bar{\theta} \cap \bar{\zeta})](x, y) .
\end{aligned}
$$

Proposition 3.13. Let $\bar{\theta}, \bar{\eta}, \bar{\zeta} \in \mathrm{FCon}(X)$. Then $\bar{\theta} \circ(\bar{\eta} \cap \bar{\zeta}) \subseteq(\bar{\theta} \circ \bar{\eta}) \cap(\bar{\theta} \circ \bar{\zeta})$. 
Proof. Assume that $\bar{\theta}, \bar{\eta}, \bar{\zeta} \in \mathrm{FCon}(X)$. Let $(x, y) \in X \times X$. Then

$$
\begin{aligned}
{[\bar{\theta} \circ(\bar{\eta} \cap \bar{\zeta})](x, y) } & =\sup _{z \in X}\{\min [\bar{\theta}(x, z),(\bar{\eta} \cap \bar{\zeta})(z, y)\} \\
& =\sup _{z \in X}\{\min [\bar{\theta}(x, z), \min \{\bar{\eta}(z, y), \bar{\zeta}(z, y)\}]\} \\
& \leq \min _{z \in X}\left\{\sup _{z \in X}\{\min [\bar{\theta}(x, z), \bar{\eta}(z, y)]\}, \sup _{z \in X}\{\min [\bar{\theta}(x, z), \bar{\zeta}(z, y)]\}\right\} \\
& =\min \{(\bar{\theta} \circ \bar{\eta})(x, y),(\bar{\theta} \circ \bar{\zeta})(x, y)\} \\
& =[(\bar{\theta} \circ \bar{\eta}) \cap(\bar{\theta} \circ \bar{\zeta})](x, y) .
\end{aligned}
$$

Definition 3.14. Let $\bar{\theta} \in \mathrm{FCon}(X)$ and $\alpha \in[0,1]$. Then the level congruence relation $\bar{\theta}^{\alpha}$ of $\bar{\theta}$ and strong level congruence $\bar{\theta}_{>}^{\alpha}$ of $X$ are defined as the following:

$$
\bar{\theta}^{\alpha}:=\{(x, y) \in X \times X: \bar{\theta}(x, y) \geq \alpha\} \text { and } \bar{\theta}_{>}^{\alpha}:=\{(x, y) \in X \times X: \bar{\theta}(x, y)>\alpha\} .
$$

Example 3.15. Consider the pseudo BE-algebra given in Example 3.2. Then

(i) if $\alpha \in(0,0.4]$, then $\bar{\theta}^{\alpha}=X \times X$,

(ii) if $\alpha \in(0.4,0.7]$, then $\bar{\theta}^{\alpha}=\Delta$, where $\Delta=\{(x, x): x \in X\}$,

(iii) if $\alpha \in(0.7,1]$, then $\bar{\theta}^{\alpha}=\varnothing$,

(iv) if $\alpha \in(0,0.4)$, then $\bar{\theta}_{>}^{\alpha}=\{(1, a),(1, b),(1, c),(a, b),(b, a),(a, c),(c, a),(c, b),(b, c)\}$,

(v) if $\alpha \in[0.4,0.7)$, then $\bar{\theta}_{>}^{\alpha}=\Delta$,

(vi) if $\alpha \in[0.7,1]$, then $\bar{\theta}_{>}^{\alpha}=\varnothing$.

Proposition 3.16. Let $\bar{\theta} \in \mathrm{FCon}(X)$ and $\alpha \in[0,1]$. Then

(i) if $\bar{\theta}^{\alpha} \neq \varnothing$, then $\bar{\theta}(1,1) \geq \alpha$,

(ii) if $\bar{\theta}^{\alpha}:=\{(x, y): \bar{\theta}(x, y)=\bar{\theta}(y, x) \geq \alpha\}$, then $\bar{\theta}^{\alpha} \neq \varnothing$ and $\bar{\theta}^{\alpha}$ is a congruence relation on $X$.

Proof. We only prove (i). Since $\bar{\theta}^{\alpha} \neq \varnothing$, there exists $(x, y) \in \bar{\theta}^{\alpha}$. Applying Lemma 3.3, we get $\bar{\theta}(1,1) \geq \bar{\theta}(x, y) \geq \alpha$.

Lemma 3.17. Let $\bar{\theta} \in \mathrm{FCon}(X)$ and $\alpha \in(0,1)$. Then

$$
\bar{\theta}^{\alpha}=\bigcap_{0 \leq t<\alpha} \bar{\theta}_{>}^{t} \text { and } \bar{\theta}_{>}^{\alpha}=\bigcup_{\alpha<t \leq 1} \bar{\theta}^{t} .
$$

Proposition 3.18. Let $\bar{\theta}$ be a fuzzy relation on $X$ and $\alpha \in(0,1)$. Then

(i) $\bar{\theta}$ is a fuzzy left (right) compatible relation if and only if $\bar{\theta}^{\alpha}\left(\bar{\theta}_{>}^{\alpha}\right)$ is a left (right) compatible relation on $X$,

(ii) $\bar{\theta}$ is a fuzzy congruence relation if and only if $\bar{\theta}^{\alpha}\left(\bar{\theta}_{>}^{\alpha}\right)$ is a congruence relation on $X$.

Proposition 3.19. Let $\bar{\theta}, \bar{\eta} \in \mathrm{FCon}(X)$ and $\alpha \in[0,1)$. Then 
(i) $\bar{\theta}=\bar{\eta}$ if and only if $\bar{\theta}_{>}^{\alpha}=\bar{\eta}_{>}^{\alpha}$,

(ii) $(\bar{\theta} \circ \bar{\eta})_{>}^{\alpha}=\bar{\theta}_{>}^{\alpha} \circ \bar{\eta}_{>}^{\alpha}$,

(iii) $\bar{\theta} \circ \bar{\eta}=\bar{\eta} \circ \bar{\theta}$ if and only if $\bar{\theta}_{>}^{\alpha} \circ \bar{\eta}_{>}^{\alpha}=\bar{\eta}_{>}^{\alpha} \circ \bar{\theta}_{>}^{\alpha}$, for all $\alpha \in[0,1)$, where, $\bar{\theta}_{>}^{\alpha} \neq \emptyset$ and $\bar{\eta}_{>}^{\alpha} \neq \emptyset$.

Proof. We only prove (i). Assume that $(x, y) \in \bar{\theta}_{>}^{\alpha}$. Then $\bar{\eta}_{>}(x, y)=\bar{\theta}_{>}(x, y)>\alpha$ and so $(x, y) \in$ $\bar{\eta}_{>}^{\alpha}$. Hence $\bar{\theta}_{>}^{\alpha} \subseteq \bar{\eta}_{>}^{\alpha}$. Similarly, $\bar{\eta}_{>}^{\alpha} \subseteq \bar{\theta}_{>}^{\alpha}$.

Conversely, let $\bar{\theta}_{>}^{\alpha}=\bar{\eta}_{>}^{\alpha}$, but there exists $(x, y) \in X \times X$ such that $\bar{\theta}(x, y) \neq \bar{\eta}(x, y)$. Let $\bar{\theta}(x, y)=t_{1}$ and $\bar{\eta}(x, y)=t_{2}$. Then $t_{1}>t_{2}$ or $t_{2}>t_{1}$. If $t_{1}>t_{2}$, then $\bar{\theta}(x, y)=t_{1}>t_{2}$, and so $(x, y) \in \bar{\theta}_{>}^{t_{1}}=\bar{\eta}_{>}^{t_{1}}$. Hence $\bar{\eta}(x, y)>t_{1}$, and so $t_{2}>t_{1}$, which is a contradiction. If $t_{2}>t_{1}$, by a similar argument we have a contradiction.

Theorem 3.20. If $\bar{\theta}$ and $\bar{\eta}$ are fuzzy left (right) compatible (congruence) relation on $X$. Then $\bar{\theta} \times \bar{\eta}$ is a left (right) compatible (congruence) relation on $X \times X$.

In this section, we investigate fuzzy congruence relations induced by fuzzy medial filters in a pseudo BE-algebra.

Theorem 3.21. Let $f$ be an endomorphism of $X$. If $\theta \in \mathrm{FCon}(X)$, then $\bar{\theta}$ is defined by $\bar{\theta}(x, y):=$ $\theta(f(x), f(y))$ is so.

Proof. It is obvious that $\bar{\theta}$ well-defined. Let $x, y, z, u \in X$.

$\left(\mathrm{FC}_{1}\right) \bar{\theta}(x, x)=\theta(f(x), f(x))=\theta(1,1)=\bar{\theta}(1,1)$.

$\left(\mathrm{FC}_{2}\right) \bar{\theta}(x, y)=\theta(f(x), f(y))=\theta(f(y), f(x))=\bar{\theta}(y, x)$.

$\left(\mathrm{FC}_{3}\right) \bar{\theta}(x, y)=\theta(f(x), f(y)) \geq \min [\theta(f(x), f(z)), \theta(f(z), f(y))]$ $=\min [\bar{\theta}(x, z), \bar{\theta}(z, y)]$.

$\left(\mathrm{FC}_{4}\right) \bar{\theta}(x \rightarrow u, y \rightarrow u)=\theta(f(x \rightarrow u), f(y \rightarrow u))$

$$
\begin{aligned}
& =\theta(f(x) \rightarrow f(u), f(y) \rightarrow f(u)) \\
& \geq \theta(f(x), f(y))=\bar{\theta}(x, y) .
\end{aligned}
$$

Similarly, $\bar{\theta}(v \rightarrow x, v \rightarrow y) \geq \bar{\theta}(x, y)$.

$$
\begin{aligned}
\left(\mathrm{FC}_{5}\right) \bar{\theta}(x \rightsquigarrow u, y \rightsquigarrow u) & =\theta(f(x \rightsquigarrow u), f(y \rightsquigarrow u)) \\
& =\theta(f(x) \rightsquigarrow f(u), f(y) \rightsquigarrow f(u)) \\
& \geq \theta(f(x), f(y))=\bar{\theta}(x, y) .
\end{aligned}
$$

Similarly, $\bar{\theta}(v \rightsquigarrow x, v \rightsquigarrow y) \geq \bar{\theta}(x, y)$.

Remark 3.22. The fuzzy subset $\bar{\theta}_{x}: X \rightarrow[0,1]$, which is defined by $\bar{\theta}_{x}(y)=\bar{\theta}(x, y)$, is called the fuzzy congruence class containing $x$.

By a routine calculation we can see that:

Proposition 3.23. Let $\bar{\theta} \in \mathrm{FCon}(X)$. Then for all $x, y, z, u \in X$

(i) $\bar{\theta}_{x}(x)=\bar{\theta}_{1}(1)=\bar{\theta}_{1}(x)$,

(ii) $\bar{\theta}_{x}(y)=\bar{\theta}_{y}(x)=\bar{\theta}_{x \rightarrow y}(1)=\bar{\theta}_{x \rightsquigarrow y}(1)$,

(iii) $\bar{\theta}_{x}(y) \geq \bar{\theta}_{x}(y \rightarrow z)$, 
(iv) $\bar{\theta}_{x}(z) \geq \min \left[\bar{\theta}_{x}(y), \bar{\theta}_{y}(z)\right]$

(v) $\bar{\theta}_{x}(z) \geq \min \left[\bar{\theta}_{x}(y), \bar{\theta}_{x}(y \rightarrow z)\right]$,

(vi) $\bar{\theta}_{x \rightarrow u}(y \rightarrow u) \geq \bar{\theta}_{x}(y)$ and $\bar{\theta}_{x \rightsquigarrow u}(y \rightsquigarrow u) \geq \bar{\theta}_{x}(y)$,

(vii) $\bar{\theta}_{u \rightarrow x}(u \rightarrow y) \geq \bar{\theta}_{x}(y)$ and $\bar{\theta}_{u \rightsquigarrow x}(u \rightsquigarrow y) \geq \bar{\theta}_{x}(y)$,

(viii) if $x \leq y$, then $\bar{\theta}_{x}(y)=\bar{\theta}_{y}(x)=\bar{\theta}_{1}(1)$,

(ix) $\bar{\theta}_{x}=\bar{\theta}_{y}$ if and only if $\bar{\theta}_{x \rightarrow z}(1)=\bar{\theta}_{y \rightarrow z}(1)$,

(x) $\bar{\theta}_{x}=\bar{\theta}_{y}$ if and only if $\bar{\theta}_{x \rightsquigarrow z}(1)=\bar{\theta}_{y \rightsquigarrow z}(1)$.

Proposition 3.24. Let $\bar{\theta} \in \mathrm{FCon}(X)$ and $x \in X$. Then $\bar{\theta}_{x}$ is a fuzzy filter of $X$.

The following example shows that the converse of Proposition 3.24 , is not valid in general.

Example 3.25. (i) ([16]) Let $X=\{a, b, c, d, 1\}$. Define the operations $\rightarrow$ and $\rightsquigarrow$ on $X$ as follows:

\begin{tabular}{c|ccccc}
$\rightarrow$ & 1 & $a$ & $b$ & $c$ & $d$ \\
\hline 1 & 1 & $a$ & $b$ & $c$ & $d$ \\
$a$ & 1 & 1 & $c$ & $c$ & 1 \\
$b$ & 1 & $d$ & 1 & 1 & $d$ \\
$c$ & 1 & $d$ & 1 & 1 & $d$ \\
$d$ & 1 & 1 & $c$ & $c$ & 1
\end{tabular}

\begin{tabular}{c|ccccc}
$\rightsquigarrow$ & 1 & $a$ & $b$ & $c$ & $d$ \\
\hline 1 & 1 & $a$ & $b$ & $c$ & $d$ \\
$a$ & 1 & 1 & $b$ & $c$ & 1 \\
$b$ & 1 & $d$ & 1 & 1 & $d$ \\
$c$ & 1 & $d$ & 1 & 1 & $d$ \\
$d$ & 1 & 1 & $b$ & $c$ & 1
\end{tabular}

Then $(X ; \rightarrow, \rightsquigarrow, 1)$ is a pseudo-BE algebra. Define $\bar{\theta}: X \times X \longrightarrow[0,1]$ as follows:

\begin{tabular}{l|lllll}
$\bar{\theta}$ & 1 & $a$ & $b$ & $c$ & $d$ \\
\hline 1 & 0.7 & 0.5 & 0.6 & 0.6 & 0.5 \\
$a$ & 0.5 & 0.7 & 0.2 & 0.3 & 0.1 \\
$b$ & 0.6 & 0.2 & 0.7 & 0.1 & 0.2 \\
$c$ & 0.6 & 0.3 & 0.1 & 0.7 & 0.4 \\
$d$ & 0.5 & 0.1 & 0.2 & 0.4 & 0.7
\end{tabular}

Then $\bar{\theta}$ is not a fuzzy congruence relation. Since

$$
\bar{\theta}(a, d)=0.1 \nsupseteq \min [\bar{\theta}(a, c), \bar{\theta}(c, d)]=\min \{0.3,0.4\}=0.3 .
$$

Routine calculations show that $\bar{\theta}_{1}(1)=0.7, \bar{\theta}_{1}(a)=\bar{\theta}_{1}(d)=0.5$ and $\bar{\theta}_{1}(b)=\bar{\theta}_{1}(c)=0.6$. It is easily seen that $\bar{\theta}_{1}: X \longrightarrow[0,1]$ is a fuzzy filter of $X$.

(ii) (4]) Let $X=\{a, b, c, d, 1\}$. Define the operations $\rightarrow$ and $\rightsquigarrow$ on $X$ as follows:

\begin{tabular}{c|ccccc}
$\rightarrow$ & 1 & $a$ & $b$ & $c$ & $d$ \\
\hline 1 & 1 & $a$ & $b$ & $c$ & $d$ \\
$a$ & 1 & 1 & $b$ & $c$ & $d$ \\
$b$ & 1 & 1 & 1 & $b$ & $c$ \\
$c$ & 1 & $a$ & 1 & 1 & $b$ \\
$d$ & 1 & $a$ & 1 & 1 & 1
\end{tabular}

\begin{tabular}{c|ccccc}
$\rightsquigarrow$ & 1 & $a$ & $b$ & $c$ & $d$ \\
\hline 1 & 1 & $a$ & $b$ & $c$ & $d$ \\
$a$ & 1 & 1 & $b$ & $c$ & $d$ \\
$b$ & 1 & 1 & 1 & $b$ & $c$ \\
$c$ & 1 & $a$ & 1 & 1 & $b$ \\
$d$ & 1 & $a$ & 1 & 1 & 1
\end{tabular}

Then $(X ; \rightarrow, \rightsquigarrow, 1)$ is a pseudo BE-algebra, but it is not distributive. Since

$$
c \rightarrow(c \rightsquigarrow d)=c \rightarrow b=1 \neq b=1 \rightsquigarrow b=(c \rightarrow c) \rightsquigarrow(c \rightarrow d) .
$$

Define a fuzzy relation $\bar{\theta}: X \times X \longrightarrow[0,1]$ as follows: 


\begin{tabular}{l|lllll}
$\bar{\theta}$ & 1 & $a$ & $b$ & $c$ & $d$ \\
\hline 1 & 0.4 & 0.2 & 0.2 & 0.2 & 0.2 \\
$a$ & 0.3 & 0.1 & 0.2 & 0.3 & 0.1 \\
$b$ & 0.1 & 0.2 & 0.2 & 0.4 & 0.1 \\
$c$ & 0.2 & 0.3 & 0.1 & 0.3 & 0.2 \\
$d$ & 0.4 & 0.1 & 0.1 & 0.4 & 0.2
\end{tabular}

Then $\bar{\theta}$ is not a fuzzy congruence relation. Since

$$
\bar{\theta}(a, d)=0.1 \nsupseteq \min [\bar{\theta}(a, c), \bar{\theta}(c, d)]=\min \{0.3,0.2\}=0.2 .
$$

Routine calculation shows that $\bar{\theta}_{1}(1)=0.4, \bar{\theta}_{1}(a)=\bar{\theta}_{1}(b)=\bar{\theta}_{1}(c)=\bar{\theta}_{1}(d)=0.2$. It is easy to see that $\bar{\theta}_{1}: X \longrightarrow[0,1]$ is a fuzzy filter of $X$.

In the following theorem we show that if $\bar{\mu}$ is a fuzzy medial filter and $X$ is distributive, then the converse of Proposition 3.24 , holds.

Theorem 3.26. Let $\bar{\mu}$ be a fuzzy medial filter in distributive pseudo BE-algebra $X$. Then there is a fuzzy congruence relation $\bar{\theta}$ in $X$ such that $\bar{\theta}_{1}=\bar{\mu}$.

Proof. Assume that $\bar{\mu}$ is a fuzzy medial filter. Define a fuzzy relation in $X$ by:

$$
\bar{\theta}(x, y)=\min [\bar{\mu}(x \rightarrow y), \bar{\mu}(y \rightarrow x)], \text { for all } x, y \in X .
$$

Then, for all $x, y \in X$, we have

$$
\begin{aligned}
\left(\mathrm{FC}_{1}\right) \bar{\theta}(x, x)=\min [\bar{\mu}(x \rightarrow x), \bar{\mu}(x \rightarrow x)] & =\min [\bar{\mu}(1), \bar{\mu}(1)] \\
& =\min [\bar{\mu}(1 \rightarrow 1), \bar{\mu}(1 \rightarrow 1)] \\
& =\bar{\theta}(1,1) .
\end{aligned}
$$

$\left(\mathrm{FC}_{2}\right) \bar{\theta}(x, y)=\min [\bar{\mu}(x \rightarrow y), \bar{\mu}(y \rightarrow x)]=\min [\bar{\mu}(y \rightarrow x), \bar{\mu}(x \rightarrow y)]$

$$
=\bar{\theta}(y, x) \text {. }
$$

$\left(\mathrm{FC}_{3}\right)$ Since $\bar{\mu}$ is a fuzzy medial filter, we have

$$
\begin{aligned}
\bar{\theta}(x, z) & =\min [\bar{\mu}(x \rightarrow z), \bar{\mu}(z \rightarrow x)] \\
& \geq \min [\min (\bar{\mu}(x \rightarrow y), \bar{\mu}(y \rightarrow z)), \min (\bar{\mu}(z \rightarrow y), \bar{\mu}(y \rightarrow x))] \\
& =\min [\min (\bar{\mu}(x \rightarrow y), \bar{\mu}(y \rightarrow x)), \min (\bar{\mu}(z \rightarrow y), \bar{\mu}(y \rightarrow z))] \\
& =\min [\bar{\theta}(x, y), \bar{\theta}(y, z)] .
\end{aligned}
$$

$\left(\mathrm{FC}_{4}\right)$ For the right compatible condition, let $u \in X$. Since

$$
\begin{aligned}
(y \rightarrow x) \rightarrow((x \rightarrow u) \rightsquigarrow(y \rightarrow u)) & =(x \rightarrow u) \rightsquigarrow((y \rightarrow x) \rightsquigarrow(y \rightarrow u)) \\
& =(x \rightarrow u) \rightsquigarrow(y \rightarrow(x \rightsquigarrow u))=1 .
\end{aligned}
$$

Thus, $(y \rightarrow x) \rightarrow((x \rightarrow u) \rightarrow(y \rightarrow u))=1$. Therefore, $\bar{\mu}((x \rightarrow u) \rightarrow(y \rightarrow u)) \geq \bar{\mu}(y \rightarrow x)$.

Similarly, we get $\bar{\mu}((y \rightarrow u) \rightarrow(x \rightarrow u)) \geq \bar{\mu}(x \rightarrow y)$. Then

$$
\begin{aligned}
\bar{\theta}(x \rightarrow u, y \rightarrow u) & =\min [\bar{\mu}((x \rightarrow u) \rightarrow(y \rightarrow u)), \bar{\mu}((y \rightarrow u) \rightarrow(x \rightarrow u))] \\
& \geq \min [\bar{\mu}(y \rightarrow x), \bar{\mu}(x \rightarrow y)] \\
& =\bar{\theta}(x, y) .
\end{aligned}
$$

By a similar argument, $\bar{\theta}(x \rightsquigarrow u, y \rightsquigarrow u) \geq \bar{\theta}(x, y)$. 
$\left(\mathrm{FC}_{5}\right)$ For the left compatible condition, let $u \in X$. Then

$$
\begin{aligned}
\bar{\theta}(u \rightarrow x, u \rightarrow y) & =\min [\bar{\mu}((u \rightarrow x) \rightarrow(u \rightarrow y)), \bar{\mu}((u \rightarrow y) \rightarrow(u \rightarrow x))] \\
& \geq \min [\bar{\mu}(x \rightarrow y), \bar{\mu}(y \rightarrow x)] \\
& =\bar{\theta}(x, y)
\end{aligned}
$$

Similarly, $\bar{\theta}(u \rightsquigarrow x, u \rightsquigarrow y) \geq \bar{\theta}(x, y)$. Also, for all $x \in X$,

$$
\bar{\theta}_{1}(x)=\bar{\theta}(1, x)=\min [\bar{\mu}(1 \rightarrow x), \bar{\mu}(x \rightarrow 1)]=\min [\bar{\mu}(x), \bar{\mu}(1)]=\bar{\mu}(x) .
$$

Therefore, $\bar{\theta}_{1}=\bar{\mu}$.

Remark 3.27. Let $\bar{\theta} \in \mathrm{FCon}(X)$. For every element $x \in X$, define:

$$
\bar{\theta}_{x}=\left\{y \in X: \bar{\theta}_{x}(y)=\bar{\theta}_{1}(1)\right\}
$$

of $X$ and $X / \bar{\theta}=\left\{\bar{\theta}_{x}: x \in X\right\}$. It is obviously that $\bar{\theta}_{x} \neq \emptyset$, for all $x \in X$ (since $x \in \bar{\theta}_{x}$ ) and $X=\bigcup_{x \in X} \bar{\theta}_{x}$. Also, define the binary operations $\rightarrow$ and $\rightsquigarrow$ on $X / \bar{\theta}$ as follows:

$$
\bar{\theta}_{x} \rightarrow \bar{\theta}_{y}=\bar{\theta}_{x \rightarrow y} \text { and } \bar{\theta}_{x} \rightsquigarrow \bar{\theta}_{y}=\bar{\theta}_{x \rightsquigarrow y} .
$$

These operations are well-defined. Because, if $\bar{\theta}_{x}=\bar{\theta}_{x^{\prime}}$ and $\bar{\theta}_{y}=\bar{\theta}_{y^{\prime}}$, then we have $\bar{\theta}\left(x, x^{\prime}\right)=$ $\bar{\theta}\left(y, y^{\prime}\right)=\bar{\theta}(1,1)$. Since

$$
\bar{\theta}(1,1)=\bar{\theta}\left(x, x^{\prime}\right) \leq \bar{\theta}\left(x \rightarrow y, x^{\prime} \rightarrow y\right) \text { and } \bar{\theta}(1,1)=\bar{\theta}\left(y, y^{\prime}\right) \leq \bar{\theta}\left(x^{\prime} \rightarrow y, x^{\prime} \rightarrow y^{\prime}\right),
$$

we have

$$
\bar{\theta}(1,1) \leq \min \left[\bar{\theta}\left(x \rightarrow y, x^{\prime} \rightarrow y\right), \bar{\theta}\left(x^{\prime} \rightarrow y, x^{\prime} \rightarrow y^{\prime}\right)\right] \leq \bar{\theta}\left(x \rightarrow y, x^{\prime} \rightarrow y^{\prime}\right) \leq \bar{\theta}(1,1) .
$$

This means that $\bar{\theta}\left(x \rightarrow y, x^{\prime} \rightarrow y^{\prime}\right)=\bar{\theta}(1,1)$ and $\bar{\theta}_{x \rightarrow y}=\bar{\theta}_{x^{\prime} \rightarrow y^{\prime}}$. By a similar argument, $\bar{\theta}_{x \rightsquigarrow y}=$ $\bar{\theta}_{x^{\prime} \rightarrow y^{\prime}}$. So, the binary operations $\rightarrow$ and $\rightsquigarrow$ are well-defined.

Theorem 3.28. If $\bar{\theta} \in \mathrm{FCon}(X)$, then $\left(X / \bar{\theta} ; \rightarrow, \rightsquigarrow, \bar{\theta}_{1}\right)$ is a pseudo BE-algebra.

Example 3.29. Consider the fuzzy congruence relation $\bar{\theta}$ given in Example 3.2 , and $1_{\bar{\theta}}=\{1\}$, $a_{\bar{\theta}}=\{a\}, b_{\bar{\theta}}=\{b\}$ and $c_{\bar{\theta}}=\{c\}$. Then $X / \bar{\theta}=\{\{1\},\{a\},\{b\},\{c\}\}$ with the following tables:

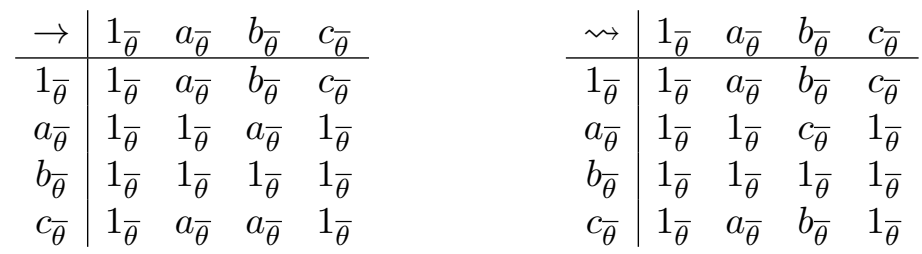

Then $\left(X / \bar{\theta} ; \rightarrow, \rightsquigarrow, 1_{\bar{\theta}}\right)$ is a pseudo BE-algebra.

Let $\bar{\theta} \in \mathrm{FCon}(X)$. The natural map of $X$ onto $X / \bar{\theta}$ is $\pi: X \longrightarrow X / \bar{\theta}$ given by $\pi(x)=\bar{\theta}_{x}$.

Remark 3.30. Assume that $\bar{\theta}, \bar{\eta} \in \mathrm{FCon}(X)$. Let $\pi_{1}: X \longrightarrow X / \bar{\theta}$ and $\pi_{2}: X \longrightarrow X / \bar{\eta}$ be the natural homomorphisms. Combining these, we obtain a homomorphism $\pi_{1} \times \pi_{2}: X \longrightarrow X / \bar{\theta} \times X / \bar{\eta}$. Then $X /(\bar{\theta} \cap \bar{\eta}) \cong X / \bar{\theta} \times X / \bar{\eta}$.

Definition 3.31. Let $\theta$ be an equivalence relation and $\bar{\theta}$ be a fuzzy relation on $X$. Then $\bar{\theta}$ is called $\theta$-invariant if $\bar{\theta}_{x}=\bar{\theta}_{a}$ and $\bar{\theta}_{y}=\bar{\theta}_{b}$ imply $\theta(x, y)=\theta(a, b)$. 
Definition 3.32. Let $\theta$ be a congruence relation and $\bar{\theta}$ be a $\theta$-invariant fuzzy relation on $X$. Define a fuzzy relation $\overline{\bar{\theta}}$ on $X / \theta$ as follows:

$$
\overline{\bar{\theta}}\left(\bar{\theta}_{x}, \bar{\theta}_{y}\right)=\bar{\theta}_{x}(y) .
$$

Proposition 3.33. Let $\bar{\theta}, \bar{\eta} \in \mathrm{FCon}(X), \bar{\theta}$ be a $\theta$-invariant, $\bar{\eta}$ be a $\eta$-invariant and $\bar{\theta} \subseteq \bar{\eta}$. Then $\overline{\bar{\theta}} \subseteq \overline{\bar{\eta}}$

Proof. Assume that $x, y \in X$. Then

$$
\overline{\bar{\theta}}\left(\bar{\theta}_{x}, \bar{\theta}_{y}\right)=\bar{\theta}_{x}(y)=\bar{\theta}(x, y) \leq \bar{\eta}(x, y)=\bar{\eta}_{x}(y)=\overline{\bar{\eta}}\left(\bar{\eta}_{x}, \bar{\eta}_{y}\right)
$$

Theorem 3.34. If $\bar{\theta}$ is a $\theta$-invariant fuzzy left (right) compatible (congruence) relation on $X$, then $\overline{\bar{\theta}}$ is so on $X / \theta$.

Proof. Since $\bar{\theta}$ is a $\theta$-invariant fuzzy left relation on $X$, we get $\overline{\bar{\theta}}$ is well defined. Let $x, y, z, u \in X$. Then

$\left(\mathrm{FC}_{1}\right) \overline{\bar{\theta}}\left(\bar{\theta}_{x}, \bar{\theta}_{x}\right)=\bar{\theta}_{x}(x)=\bar{\theta}_{1}(1)$, on the other hand, $\overline{\bar{\theta}}\left(\bar{\theta}_{1}, \bar{\theta}_{1}\right)=\bar{\theta}_{1}(1)$. So, $\overline{\bar{\theta}}\left(\theta_{x}, \theta_{x}\right)=\bar{\theta}\left(\bar{\theta}_{1}, \bar{\theta}_{1}\right)$.

$\left(\mathrm{FC}_{2}\right) \overline{\bar{\theta}}\left(\bar{\theta}_{x}, \bar{\theta}_{y}\right)=\bar{\theta}(x, y)=\bar{\theta}(y, x)=\overline{\bar{\theta}}\left(\bar{\theta}_{y}, \bar{\theta}_{x}\right)$

$\left(\mathrm{FC}_{3}\right) \overline{\bar{\theta}}\left(\bar{\theta}_{x}, \bar{\theta}_{y}\right)=\bar{\theta}(x, y) \geq \min [\bar{\theta}(x, z), \bar{\theta}(z, y)]=\min \left[\overline{\bar{\theta}}\left(\bar{\theta}_{x}, \bar{\theta}_{z}\right), \overline{\bar{\theta}}\left(\bar{\theta}_{z}, \bar{\theta}_{y}\right)\right]$.

$\left(\mathrm{FC}_{4}\right) \overline{\bar{\theta}}\left(\bar{\theta}_{x} \rightarrow \bar{\theta}_{u}, \bar{\theta}_{y} \rightarrow \bar{\theta}_{u}\right)=\overline{\bar{\theta}}\left(\bar{\theta}_{x \rightarrow u}, \bar{\theta}_{y \rightarrow u}\right)=\bar{\theta}(x \rightarrow u, y \rightarrow u)$

$$
\geq \bar{\theta}(x, y)=\overline{\bar{\theta}}\left(\bar{\theta}_{x}, \bar{\theta}_{y}\right) \text {. }
$$

By a similar argument, $\overline{\bar{\theta}}\left(\bar{\theta}_{u} \rightarrow \bar{\theta}_{x}, \bar{\theta}_{u} \rightarrow \bar{\theta}_{y}\right) \geq \overline{\bar{\theta}}\left(\bar{\theta}_{x}, \bar{\theta}_{y}\right)$.

$$
\begin{aligned}
\left(\mathrm{FC}_{5}\right) \overline{\bar{\theta}}\left(\bar{\theta}_{x} \rightsquigarrow \bar{\theta}_{u}, \bar{\theta}_{y} \rightsquigarrow \bar{\theta}_{u}\right)=\overline{\bar{\theta}}\left(\bar{\theta}_{x \rightsquigarrow u}, \bar{\theta}_{y \rightsquigarrow u}\right) & =\bar{\theta}(x \rightsquigarrow u, y \rightsquigarrow u) \\
& \geq \bar{\theta}(x, y) \\
& =\overline{\bar{\theta}}\left(\bar{\theta}_{x}, \bar{\theta}_{y}\right)
\end{aligned}
$$

Similarly, $\overline{\bar{\theta}}\left(\bar{\theta}_{u} \rightsquigarrow \bar{\theta}_{x}, \bar{\theta}_{u} \rightsquigarrow \bar{\theta}_{y}\right) \geq \overline{\bar{\theta}}\left(\bar{\theta}_{x}, \bar{\theta}_{y}\right)$. Also, assume that $\overline{\bar{\theta}}_{\bar{\theta}_{x}}=\overline{\bar{\theta}}_{\bar{\theta}_{a}}$ and $\overline{\bar{\theta}}_{\bar{\theta}_{y}}=\overline{\bar{\theta}}_{\bar{\theta}_{b}}$, for some $a, b \in X$. Then $\overline{\bar{\theta}}_{\bar{\theta}_{x}}\left(\bar{\theta}_{c}\right)=\overline{\bar{\theta}}_{\bar{\theta}_{a}}\left(\bar{\theta}_{c}\right)$ and $\overline{\bar{\theta}}_{\bar{\theta}_{y}}\left(\bar{\theta}_{c}\right)=\overline{\bar{\theta}}_{\bar{\theta}_{b}}\left(\bar{\theta}_{c}\right)$, for all $c \in X$. Hence $\bar{\theta}_{x}(c)=\bar{\theta}(x, c)=$ $\bar{\theta}(a, c)=\bar{\theta}_{a}(c)$ and $\bar{\theta}_{y}(c)=\bar{\theta}(y, c)=\bar{\theta}(b, c)=\bar{\theta}_{b}(c)$, and so $\bar{\theta}_{x}=\bar{\theta}_{a}$ and $\bar{\theta}_{y}=\bar{\theta}_{b}$. Since $\bar{\theta}$ is $\theta$ invariant fuzzy relation, we have $\theta(x, y)=\theta(a, b)$. Therefore, $\overline{\bar{\theta}}$ is a $\theta$-invariant fuzzy relation.

\section{Conclusions}

A fuzzy congruence relation is a generalization of a congruence relation on an algebraic structure. In this paper, we introduced the notion of the fuzzy congruence relation on a pseudo BE-algebra and investigated some of their properties. Moreover, we have showed that $(\operatorname{FCon}(X), \subseteq)$ is a modular lattice. Also, fuzzy congruence relation derived from a fuzzy medial filter is investigated.

As future work, the relation between fuzzy congruence relations and fuzzy homomorphisms will be study. Also, the fuzzy homomorphism theorems an extension of homomorphism theorems can be investigated. 


\section{References}

[1] A. Borumand Saeid, A. Rezaei, R.A. Borzooei, Some types of filters in BE-algebras, Mathematics in Computer Science, 7(3) (2013), 341-352.

[2] R.A. Borzooei, M. Bakhsi, T-fuzzy congruences and T-fuzzy filters of a BL-algebra, Iranian Journal of Fuzzy Systtems, 6(4) (2009), 37-47.

[3] R.A. Borzooei, A. Borumand Saeid, A. Rezaei, A. Radfar, R. Ameri, On pseudo BE-algebras, Discussiones Mathematica, General Algebra and Applications, 33 (2013), 95-108.

[4] R.A. Borzooei, A. Borumand Saeid, A. Rezaei, A. Radfar, R. Ameri, On distributive pseudo BE-algebras, Fasciculi Mathematici, 54 (2015), 21-39.

[5] L.C. Ciungu, Commutative pseudo BE-algebras, Iranian Journal of Fuzzy Systtems, 13(1) (2016), 131-144.

[6] G. Dymek, A. Walendziak, Fuzzy ideals of pseudo BCK algebras, Demonstratio Mathematica, XLV, 1 (2012), 1-15.

[7] H.S. Kim, Y.H. Kim, On BE-algebras, Scientiae Mathematicae Japonicae, 66(1) (2007), 113117.

[8] M. Kondo, Fuzzy congruences on groups, Quasigroups and Related Systems, 11 (2004), 59-70.

[9] K.J. Lee, Fuzzy ideals of pseudo BCI-algebras, Journal of Applied Mathematics and Informatics, 27 (2009), 795-807.

[10] V. Murali, Fuzzy congruence relations, Fuzzy Sets and Systems, 30 (1989), 155-163.

[11] V. Murali, Fuzzy congruence relations, Fuzzy Sets and Systems, 41 (1991), 359-369.

[12] A. Rezaei, Fuzzy medial filters on pseudo BE-algebras, Submitted.

[13] A. Rezaei, A. Borumand Saeid, On fuzzy subalgebras of BE-algebras, Afrika Matematika, 22(2) (2011), 115-127.

[14] A. Rezaei, A. Borumand Saeid, Fuzzy congruence relations in CI-algebras, Neural Computing and Applications, 21(1) (2012), 319-328.

[15] A. Rezaei, A. Borumand Saeid, A. Radfar, R.A. Borzooei, Congruence relations on pseudo BE-algebras, Annals of the University of Craivova, Mathematics and Computer Science Series, 41(2) (2014), 166-176.

[16] A. Walendziak, A. Rezaei, Fuzzy filters of pseudo BE-algebras, Afrika Matematika, (on line), (2019), 1-12.

[17] A. Walendziak, M. Wojciechowska-Rysiawa, Fuzzy ideals of pseudo BCH-algebras, Mathematica Aeterna, 5(5) (2015), 867-881.

[18] L.A. Zadeh, Fuzzy sets, Information and Control, 8 (1965), 338-353. 